\title{
Triple crystal disease: monosodium urate monohydrate, calcium pyrophosphate dihydrate, and basic calcium phosphate in a single joint
}

\author{
PAUL B HALVERSON AND LAWRENCE M RYAN \\ From the Division of Rheumatology, Medical College of Wisconsin, Milwaukee, Wisconsin, USA
}

SUMMARY A 49 year old man is described with a polyarticular arthritis. Synovial fluid aspirate from the knee joint showed monosodium urate monohydrate and calcium pyrophosphate dihydrate by polarised light microscopy. Additionally, diphosphonate binding and scanning electron microscopy with energy dispersive analysis showed that basic calcium phosphate crystale were also present. This appears to be the first report of three crystals occurring simultaneously i a single joint.

Key word: arthritis.

The simultaneous occurrence of two different crystal species in synovial fluid has long been recognised for monosodium urate monohydrate (MSU) and calcium pyrophosphate dihydrate (CPPD) crystals. ${ }^{1}$ More recently Dieppe and his colleagues have coined the term 'mixed crystal deposition disease' to describe the occurrence of hydroxyapatite and calcium pyrophosphate crystals. ${ }^{2}$ We report here the first observation of three crystal species-MSU, CPPD, and basic calcium phosphate $(\mathrm{BCP})$-in one knee joint.

\section{Case report}

A 49 year old bricklayer was first seen in the rheumatology clinic at the Milwaukee Regional Medical Center in February 1980. He had a history of 12 years of attacks of gout, occurring approximately twice annually and proved by the observation of negatively birefringent crystals in synovial fluid. These attacks had occurred in the right first metatarsophalangeal joint, both ankles, both knees,

Accepted for publication 20 November 1987.

Correspondence to Dr Paul B Halverson, Medical College of Wisconsin, $8700 \mathrm{~W}$ Wisconsin Avenue, Milwaukee, Wisconsin 53226, USA. both elbows, and the right shoulder. The patient had received intra-articular corticosteroif injections into his knees in the past but did not know the number. Drug treatment included colchicine foop acute attacks and intermittent use of allopurinol and indomethacin, determined in part by his ability to buy these drugs. His history was also remarkable for considerable alcohol intake of at least seven beers day and a six month history of hypertension, which was not being treated.

Physical examination showed an obese man in no distress. Blood pressure was $188 / 120 \mathrm{mmHg}$ Tophaceous deposits were present in the pinna of the right ear, over the extensor surfaces of the elbows and the Achilles tendons. There were 15-2 degree flexion contractures of the elbows. Osteophyte formation was present in the right fourti distal interphalangeal joint. Flexion contractures $\phi 6$ 10 and 5 degrees were present in the right and lef knees, and range of motion was further reduced by flexion only to $100-110$ degrees. Knees were warm and effusions were present bilaterally. Ankle showed mild loss of flexion and extension. The metatarsophalangeal joints were tender, but no synovitis was detected.

Radiographs of the knees showed periarticulat demineralisation, meniscal calcifications, subchoof 
dral bone cysts, and osteophyte formation on the tibial spines and plateau without joint space narrowing. Ankle films showed extensive osteophytosis of the tibiotalar articulation.

Laboratory evaluation was as follows: uric acid 0.4 and $0.49 \mathrm{mmol} / \mathrm{l}$, calcium 2.7 and $2.6 \mathrm{mmol} / \mathrm{l}$, phosphorus $1.0 \mathrm{mmol} / \mathrm{l}$, iron $10.7 \mu \mathrm{mol} / \mathrm{l}$, total iron binding capacity $64.4 \mu \mathrm{mol} / 1$, magnesium $1.1 \mathrm{mmol} / \mathrm{l}$, thyroxine (T4) $69.5 \mathrm{nmol} / \mathrm{l}$ (normal 64.4-148.0), triiodothyronine (T3) resin uptake 0.33 (normal $0.25-0.35$ ), free thyroxine index 1.78 (normal $1 \cdot 2-4$ ), and parathyroid hormone 45 microlitre equivalents $/ \mathrm{ml}$ (normal 20-70). Synovial fluid was aspirated from the right knee. The total leucocyte cell count was $2 \cdot 75 \times 10^{9} / 1$ with $71 \%$ polymorphonuclear leucocytes. Compensated polarised light microscopy showed many negatively birefringent needle shaped crystals and rare weakly positive birefringent rhomboidal crystals. $\left[{ }^{14} \mathrm{C}\right]$ Ethane-1hydroxy-1,1-diphosphonate binding on $1 \mathrm{ml}$ of fluid was $7.9 \%$, equivalent to binding of $5 \mu \mathrm{g} / \mathrm{ml}$ of hydroxyapatite standard. Scanning electron microscopy of the washed pellet demonstrated microspheroidal particles, and energy dispersive analysis showed a calcium to phosphorus molar ratio of $1 . \overline{1}$ (SD $0 . \overline{24}$ ), compatible with a mixture of hydroxyapatite and octacalcium phosphate.

\section{Discussion}

Identification of crystals in synovial fluids has permitted classification of several rheumatic diseases and suggests pathogenetic relations between crystals and disease processes. When more than one crystal is present the interrelationships are not so easily determined. It is possible that factors which favour deposition of one crystal may also favour deposition of others. Dieppe and Watt observed MSU, CPPD, and BCP crystals in several patients, but these were not all found in the same joint. ${ }^{3}$ They suggested that there might be a deficiency in factors which inhibit crystal formation.

In the case presented here MSU, CPPD, and BCP (partially carbonate substituted hydroxyapatite, octacalcium phosphate, and rarely tricalcium phosphate) crystals were detected simultaneously in one knee joint. We suggest the term 'triple crystal disease' to describe this phenomenon. The synovial leucocyte count was slightly raised. This was probably related to the MSU crystals as BCP crystals and low levels of CPPD crystals are not usually associated with synovial leucocyte increase. Knee radiographs showed chondrocalcinosis, osteophyte formation, and subchondral bone cysts. The tibiotalar osteophytes, elbow flexion contractures, and degenerative changes in the hand suggest a generalised arthropathy.

Hyperuricaemia may have been related to chronic alcohol ingestion. Although the patient did have one raised serum calcium value, a repeat calcium was normal, and the parathyroid hormone level was not raised. Iron studies did not suggest haemochromatosis.

It is difficult to assign responsibility for the clinical picture observed to a single crystal when multiple crystals are present. The inflammatory potential of MSU and CPPD are well known. The pathogenetic significance of $\mathrm{BCP}$ crystals is less clear. Basic calcium phosphate crystals have been found in osteoarthritis and are related to the severity of joint degeneration. ${ }^{45}$ Recent reports suggest that BCP and CPPD crystals are more likely to occur together than either occur alone and that CPPD crystals tend to correlate with age, whereas BCP crystals appear to correlate with joint degeneration. ${ }^{56}$ Thus degeneration in joints, which has been associated with CPPD deposition, may actually be more closely associated with BCP deposition.

\section{References}

1 Skinner M, Cohen A S. Calcium pyrophosphate dihydrate deposition disease. Arch Intern Med 1969; 123: 636-44.

2 Dieppe P A, Doyle D V, Huskisson E C, Willoughby D A, Crocher P R. Mixed crystal deposition disease and osteoarthritis. $B r$ Med J 1978; i: 150.

3 Dieppe P, Watt I. Crystal deposition in osteoarthritis: an opportunistic event? Clin Rheum Dis 1985; 11: 367-92.

4 Halverson P B, McCarty D J. Identification of hydroxyapatite crystals in synovial fluid. Arthritis Rheum 1979; 22: 389-95.

5 Gibilisco P A, Schumacher H R, Hollander J L, Soper K A. Synovial fluid crystals in osteoarthritis. Arthritis Rheum 1985; 28: 511-5.

6 Halverson P B, McCarty D J. Patterns of radiographic abnormalities associated with basic calcium phosphate and calcium pyrophosphate dihydrate crystal deposition in the knee. Ann Rheum Dis 1986; 45: 603-5. 